\title{
Enhanced external counterpulsation in ischemic heart disease and congestive heart failure
}

$\mathrm{H}$ nhanced external counterintroduced in the 1960s as a noninvasive procedure for the treatment of angina refractory to medical therapy. Since its inception, technological advances have facilitated the procedure and rekindled interest in EECP as a means to treat symptomatic coronary artery disease and heart failure.

The principle of EECP is simple: mechanically increase venous return to the heart and decrease cardiac afterload. The procedure itself is similarly straightforward: 3 compressive cuffs are wrapped around each leg and synchronized to inflate during cardiac diastole (Fig. 1); a

I retrograde pulse wave is created that increases venous return to the heart, thereby increasing coronary artery perfusion pressure; deflation of the cuffs at the onset of cardiac systole creates negative pressure from the peripheral arterial system that de- creases cardiac afterload. The hemodynamic effect of EECP (Fig. 2) is similar to that of an intra-aortic balloon pump. By promoting venous return and decreasing afterload, EECP can decrease oxygen consumption and enhance cardiac output by up to $25 \%$. Patients usually undergo 35 consecutive 1-hour sessions of EECP over 5-7 weeks.

\section{EECP in chronic stable angina}

There is evidence that patients with chronic stable angina treated with EECP have less angina, improved exercise tolerance, decreased nitrate use and improved quality of life. Data from the International EECP Patient Registry indicate that $69 \%$ of patients improved by at least 1 Canadian Cardiovascular Society (CCS) angina class immediately after EECP; of these patients, $72 \%$ had sustained improvement at 1-year follow-up. Those with the most severe coronary artery disease and those who had previously undergone a surgical revascularization procedure $(89 \%$ of patients in the registry) seemed to benefit the most.

Only one randomized controlled trial investigating EECP for the treatment of angina has been reported to date. ${ }^{2}$ In this trial 139 patients with documented evidence of coronary artery disease, CCS angina class I-III and a positive exercise treadmill test result for ischemia were recruited. Patients in the active treatment group had a significant increase in time to STsegment depression during exercise and decrease in anginal symptoms. However, there was no difference in exercise duration, angina count and nitroglycerin use between the treatment and control groups. Adverse events such as leg pain and skin abrasions were more frequent in the treatment group than in the control group.

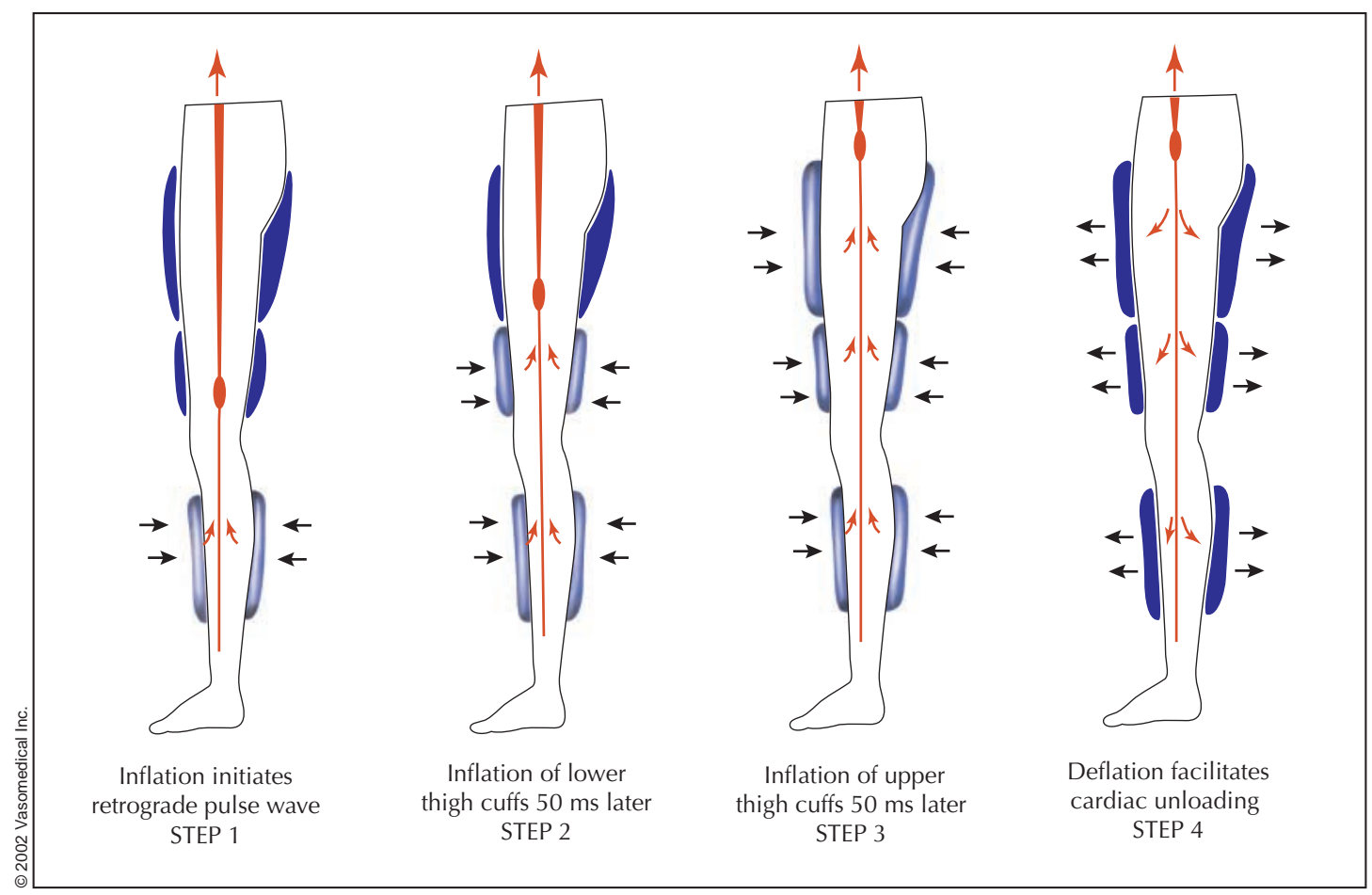

Fig. 1: Schematic of enhanced external counterpulsation (EECP), showing sequential cuff inflation at the onset of diastole to raise diastolic pressure, and of deflation just before cardiac systole to lower systolic pressure. 
How would EECP confer long-term benefits? A number of factors may play a role. Aside from the improved coronary perfusion and left ventricular systolic unloading that occurs during a treatment session, EECP is associated with favourable biochemical effects. For example, increased blood nitric oxide levels have been shown 1 week following EECP. Nitric oxide has important vasodilatory, antiplatelet, antithrombotic and antiinflammatory properties, all of which contribute to improved vascular endothelial function. Also, the shear forces induced by EECP may influence arteriogenesis and angiogenesis by upregulating the production of growth factors such as vascular endothelial growth factor and platelet-derived growth factor.

\section{EECP in heart failure}

The experience with EECP in treating heart failure is even more limited than that in treating ischemic heart disease. $\mathrm{Pa}$ tients with heart failure were initially excluded from studies because of a theoretical risk of exacerbating heart failure - it was felt that the increased venous return might overload the weakened left ventricle or precipitate ischemia by increasing stress on the ventricle wall. However, experimental studies demonstrated increased coro-

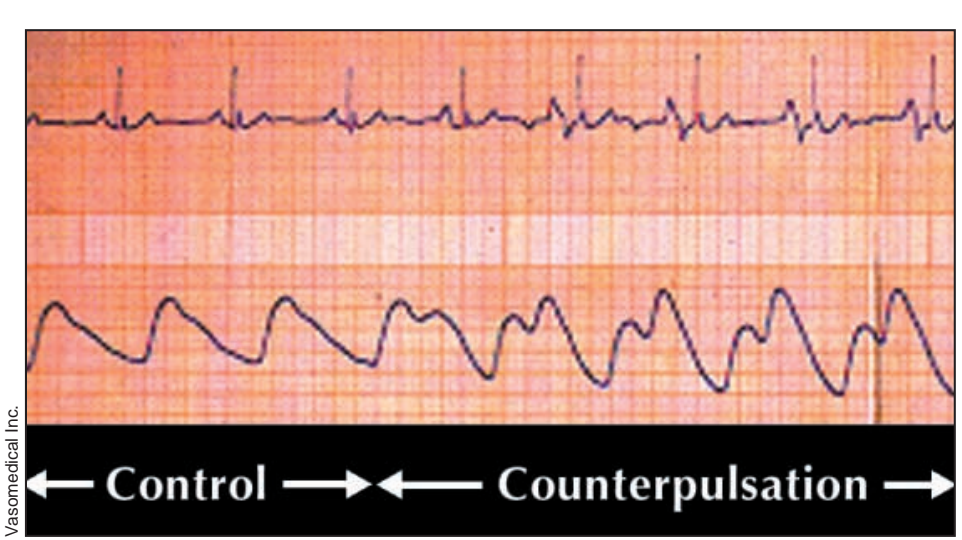

Fig. 2: Evidence of hemodynamic effect of EECP. Tracing resembles that of a patient with an intra-aortic balloon pump.

nary perfusion, increased intrinsic myocardial contractility, improved left ventricular diastolic pressure and decreased cardiac work after EECP. ${ }^{1}$

Results from an early clinical trial of EECP involving patients with angina and heart failure suggested that, among patients treated with EECP, those who had both angina and heart failure had more heart failure, more hospital admissions and higher mortality than patients who had angina alone. ${ }^{3}$ Conversely, a recently published clinical trial demonstrated improved exercise tolerance and quality of life after EECP for patients with New York Heart Association class II-III heart failure (average ejection fraction of $23 \%$ ) secondary to ischemic or dilated cardiomyopathy. There were no significant adverse events reported. ${ }^{4}$ Furthermore, data from the International EECP Patient Registry show that that patients with reduced left ventricular function $(<35 \%)$ achieved similar reductions in angina as those with preserved ejection fraction. ${ }^{3}$ Despite these promising results, the current evidence is limited to a subset of patients in a registry and small case series; the lack of appropriate controls and small samples limit the generalizability of the results. These results will need to be verified by a larger, prospective randomized controlled trial currently in progress. ${ }^{3}$

\section{Conclusion}

Although preliminary results are encouraging, the role of EECP in ischemic heart disease and heart failure will need to be elucidated by larger trials that are underway. The American College of Cardiology and the American Heart Association currently state that additional data from clinical trials are needed before they can make definitive recommendations on the use of EECP for chronic stable angina; they also conclude that there is insufficient evidence on the effectiveness and safety of EECP to advocate its use in patients with chronic heart failure.

The cost is currently estimated to be US\$5000-\$7000 for a 35-treatment session. However, if found to be effective and safe, EECP could result in an enormous cost savings by reducing the amount of medication patients require and the occurrence of adverse events secondary to medication interactions that often result in hospital admissions.

\section{Lisa Mielniczuk}

Luís Beck da Silva

Haissam Haddad

University of Ottawa Heart Institute Ottawa, Ont.

\section{References}

1. Arora RR, Chou TM, Jain D, Fleishman B, Crawford L, McKiernan T, et al. The multicenter study of enhanced external counterpulsation (MUSTEECP): effect of EECP on exerciseinduced myocardial ischemia and anginal episodes. $7 \mathrm{Am}$ Coll Cardiol 1999;33(7):1833-40.

2. Linnemeier G, Rutter MK, Barsness G, Kennard ED, Nesto RW; IEPR Investigators. Enhanced external counterpulsation for the relief of angina in patients with diabetes: safety, efficacy and 1 -year clinical outcomes. $\mathrm{Am}$ Heart 7 2003;146(3):453-8.

3. Bonetti PO, Holmes DR, Lerman A, Barsness GW. Enhanced external counterpulsation for ischemic heart disease. What's behind the curtain? 7 Am Coll Cardiol 2003;41(11):1918-25.

4. Soran O, Fleishman B, Demarco T, Grossman W, Schneider VM, Manzo $\mathrm{K}$, et al. Enhanced external counterpulsation in patients with heart failure: a multicenter feasibility study. Congest Heart Fail 2002;8(4):204-8,227. 